\section{Cils et morphogenèse cardiaque}

Nicolas Diguet, Sigolène M. Meilhac

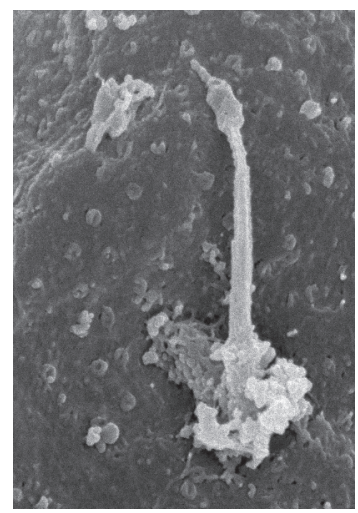

Institut Pasteur, Département de biologie du développement et cellules souches, 25, rue du Docteur Roux, 75015 Paris, France; CNRS URA2578, 75015 Paris, France.

sigolene.meilhac@pasteur.fr

(Figure 1A), et les cils primaires non motiles, qui ont une fonction sensorielle et participent à la transduction de signaux (Figure IB-C). Si la structure des cils est connue depuis plus d'un siècle, c'est en 2000 que G.J. Pazour et al. ont démontré pour la première fois le rôle fondamental des cils primaires dans le développement embryonnaire [2]. Les maladies humaines résultant d'un dysfonctionnement des cils - les ciliopathies - conduisent à des syndromes pléiotropes, ce qui indique que les cils sont nécessaires au développement d'un grand nombre d'organes. Cependant, un dysfonctionnement des cils peut conduire à des phénotypes opposés, tels qu'une hypoplasie (déficit de cellules) ou une mégalie (excès de croissance). Cela souligne l'intérêt de comprendre la fonction des cils dans un contexte particulier, en termes de type cellulaire et de stade du développement. Dans cette synthèse, nous nous intéresserons au rôle des cils dans la formation du cœur, et aux voies de signalisation dont la transduction est potentiellement assurée dans les cils cardiaques. Nous discuterons les données issues des lignées de souris génétiquement modifiées, qui apportent un éclairage nouveau sur les mécanismes potentiellement impliqués dans les cardiopathies associées aux ciliopathies.

\section{Ciliopathies et cardiopathies}

Les cils sont des organites présents dans la plupart des cellules. Ce sont des protrusions membranaires qui se développent sur un squelette de microtubules (l'axonème) ancré sur un centriole basal [45] $(\rightarrow)$. $\rightarrow$ Voir la Synthèse de C. Fort et P. Bastin, page 955 de ce numéro Les cils fonctionnent comme des antennes, intégrant des signaux mécaniques et moléculaires [1]. On distingue les cils motiles, capables de générer des flux de liquide

Cet article fait partie du numéro thématique de médecine/sciences intitulé «Cils primaires et ciliopathies $\gg\left(\mathrm{m} / \mathrm{s} \mathrm{n}^{\circ} 11\right.$, vol. 30 , novembre 2014).
Les ciliopathies sont des maladies rares pour lesquelles plusieurs variations génétiques ont déja été identifiées [3] ; elles sont classées en R. Bachmann-Gagescu, page 1011 de ce numéro différents syndromes, selon leurs manifestations cliniques [46] $(\rightarrow)$. Ces maladies graves peuvent être associées à des malformations cardiaques (Tableau I), avec des conséquences importantes sur la qualité et la durée de vie des patients. Près de $10 \%$ des patients atteints du syndrome de Bardet-Biedl [47] $(\rightarrow)$ présentent des malformations du cœur, $(\rightarrow)$ Voir la Synthèse de K. Chennen et al., page 1034 de ce numéro 

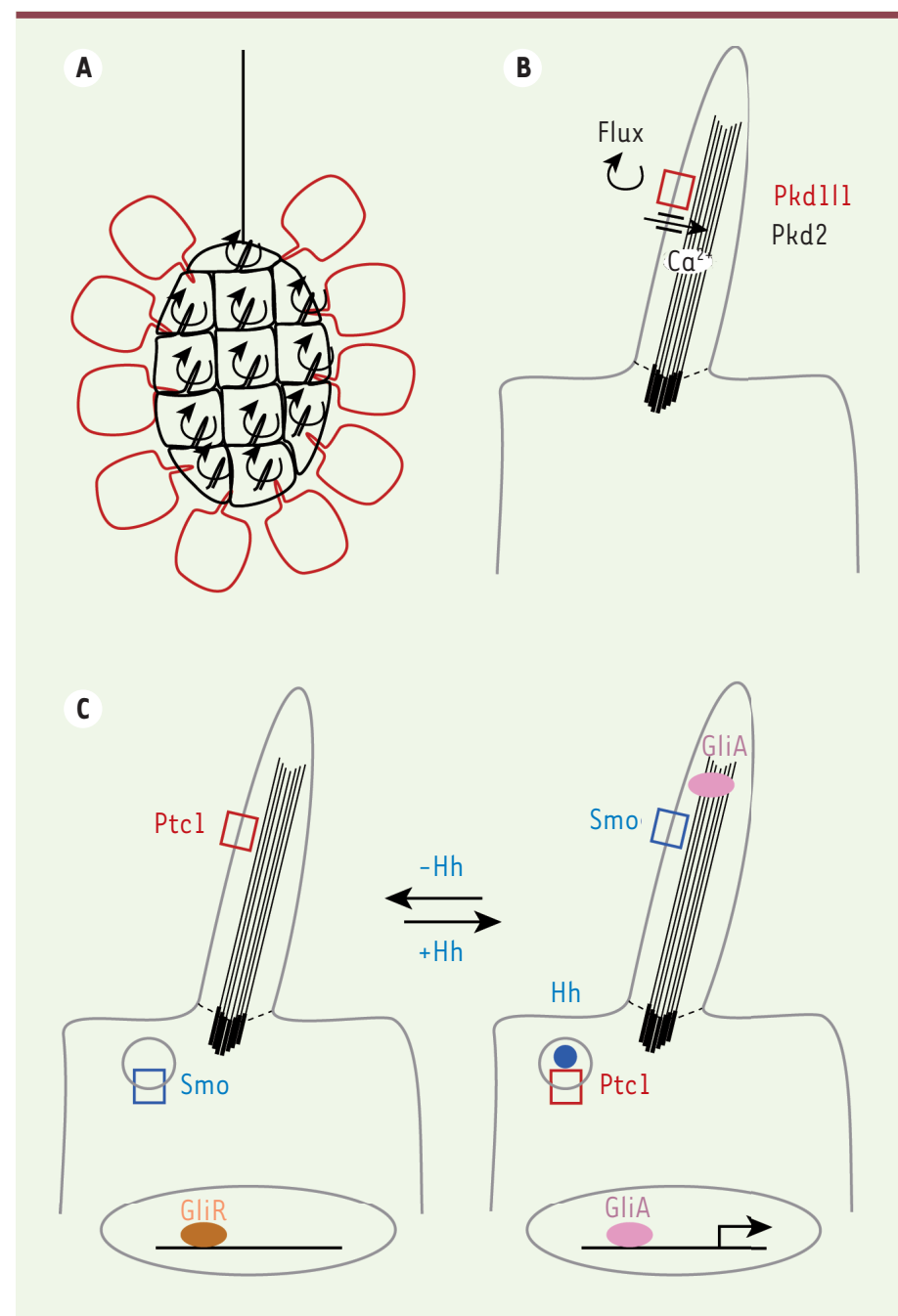

Figure 1. Structure des cils et transduction de signaux. A. Dans le nœud, les cellules du centre (en noir) possèdent des cils motiles qui génèrent un flux vers la gauche de l'embryon. Dans les cellules périphériques (en rouge), des cils primaires perçoivent ce signal. B. Les cils primaires de ces cellules sont riches en polycystines, Pkd1ll et Pkd2. Les cils primaires sont des protrusions de membrane plasmique (en gris) construites sur un squelette de microtubules (rayures noires) ancré sur un centriole basal (rayures épaisses). Les cils sont isolés du cytosol par un filtre (ligne pointillée). C. Dans d'autres types cellulaires, potentiellement les cellules précurseurs des cloisons du cœur, les cils primaires sont requis pour la transduction de la voie Hedghog (Hh). En l'absence de signal (à gauche), le récepteur inhibiteur Patched-1 (Ptcl) est localisé dans le cil, alors que le co-récepteur Smoothened (Smo) est internalisé dans la cellule. Les facteurs de transcription Gli sont sous leur forme répressive (GliR). En présence de signal (à droite), le récepteur inhibiteur Ptcl est internalisé et le co-récepteur Smoothened (Smo) peut s'accumuler dans le cil. Ceci permet l'activation des facteurs de transcription Gli (GliA).

telles que des sténoses valvulaires, une bicuspidie de la valve aortique ou des défauts de cloisonnement des oreillettes. Les patients atteints des syndromes de Meckel (OMIM 249000) ou de McKusick-Kaufman (OMIM236700), causés par des mutations dans des protéines requises pour la ciliogenèse, présentent parfois des malformations cardiaques. Le syndrome d'Alström (0MIM203800), que caractérisent plus de 80 mutations différentes dans le seul gène ALMSI (Alström syndrome 1) de fonction inconnue, est associé à une cardiomyopathie dilatée chez les deux tiers des patients. Récemment, il a été suggéré que des mutations d'ALMSI étaient responsables de cardiomyopathies avec une forte activité mitogénique [4]. Le syndrome Ellis-van Creveld ( $(V C)$, dû à des mutations du gène $E V C$ ou $E V C 2$, est associé à des défauts de cloisonnement du cœur dans $60 \%$ des cas, se traduisant par une oreillette commune ou un canal atrio-ventriculaire. La polykystose rénale autosomique dominante $(P K D)$ résulte de mutations dans les polycystines PKDI (polycystic kidney disease 1) et PKD2, des protéines souvent localisées dans le cil primaire. PKD est ainsi considérée comme une ciliopathie dans laquelle les cils sont présents, mais moins fonctionnels $[5,48](\rightarrow)$.

$(\rightarrow)$ Voir la Synthèse de M. Faces-Passy, page 1024 de ce numéro Les patients souffrant de PKD ont une probabilité accrue de développer des anomalies des valves cardiaques et une hypertrophie du ventricule gauche.

D'autres types de ciliopathies, affectant d'autres cils que les cils primaires, peuvent s'exprimer par des malformations cardiaques. Il s'agit des dyskinésies ciliaires qui affectent les cils motiles requis pour la détermination de la droite et de la gauche de l'organisme [6]. Le syndrome de Kartagener correspond à un situs inversus dans lequel la position du cœur est inversée, à droite de la cavité thoracique. Dans les autres cas, la détermination de la droite et de la gauche est incomplètement inversée, ce qui cause un spectre de malformations cardiaques regroupées sous le terme d'hétérotaxie [7], incluant une transposition des gros vaisseaux, un ventricule droit à double issue (Figure 2), un isomérisme des oreillettes et un canal atrio-ventriculaire. La survenue de malformations cardiaques associées à un dysfonctionnement des cils amène à se demander dans quel type cellulaire les cils sont requis pour la formation du cœur et quels sont les mécanismes impliqués.

\section{Localisation des cils primaires dans le cœur}

Le cil primaire est un organite présent dans de nombreux types cellulaires, en dehors de la phase de mitose puisqu'il réquisitionne le centriole pour son ancrage. Dans les cellules des différents tissus cardiaques, des observations par microscopie électronique ont établi la présence de cils primaires, c'està-dire non motiles car dépourvus d'un doublet central de microtubules (Figure $3, \varepsilon 16,5)[45](\rightarrow)$. $(\rightarrow)$ voir la synthèse page 955 de ce numéro 


\begin{tabular}{|c|c|c|c|c|c|}
\hline & Syndromes & Gènes mutés & Cardiopathies & $\begin{array}{l}\text { Référence } \\
\text { OMIM }\end{array}$ & $\begin{array}{l}\text { Prévalence } \\
\text { (source } \\
\text { Orphanet) }\end{array}$ \\
\hline \multirow[t]{2}{*}{$\begin{array}{l}\text { Cil } \\
\text { motile }\end{array}$} & $\begin{array}{l}\text { Dyskinésie ciliaire } \\
\text { primitive }\end{array}$ & $\begin{array}{l}\text { ARMC4, C210RF59, } \\
\text { CCDC39/40/65/103/114, } \\
\text { DNAAF1/2/3, DNAH5/11, } \\
\text { DNAI1/2, DNAL1, DRC1, } \\
\text { DYXIC1, HEATR2, HYDIN, } \\
\text { LRRC6, NME8, OFD1, RPGR, } \\
\text { RSPH1/4A/9, SPAG1, ZMYND10 }\end{array}$ & $\begin{array}{l}\text { Communication interauriculaire, } \\
\text { transposition des gros vaisseaux, } \\
\text { hétérotaxie }\end{array}$ & 244400 & $1 / 20000$ \\
\hline & Kartagener & DNAII & Situs inversus (dextrocardia) & 244400 & \\
\hline \multirow{6}{*}{$\begin{array}{l}\text { Cil } \\
\text { primaire }\end{array}$} & Alström & ALMSI & Cardiomyopathie dilatée & 203800 & 450 cas au monde \\
\hline & Ellis-van Creveld & $\varepsilon V C, \varepsilon V C 2$ & Communication inter-auriculaire & 225500 & 150 cas au monde \\
\hline & $\begin{array}{l}\text { Polykystose rénale } \\
\text { autosomique } \\
\text { dominante }\end{array}$ & PKDI-2 & $\begin{array}{l}\text { Hypertrophie du ventricule gauche, } \\
\text { prolapsus de la valve mitrale }\end{array}$ & 173900 & $1 / 1000$ \\
\hline & McKusick-Kaufman & MKKS & Malformations cardiaques & 236700 & $\begin{array}{l}1 \% \text { dans la } \\
\text { population Amish }\end{array}$ \\
\hline & Bardet-Biedl & $B B S 1-19$ & Malformations cardiaques & 209900 & $\begin{array}{l}1 / 125000 \text { à } \\
1 / 175000\end{array}$ \\
\hline & Meckel-Gruber & $\begin{array}{l}\text { MKSI-11, IFT88, RPGRIPI, } \\
\text { BBS15 }\end{array}$ & Malformations cardiaques & 249000 & $\begin{array}{l}1 / 9000 \\
\text { en Finlande }\end{array}$ \\
\hline
\end{tabular}

Tableau I. Cardiopathies associées à des ciliopathies. Fonctions associées aux produits des gènes cités : ARMC4, CCDC39/65/103/114, DNAAF2/3, DYXICl, HEATR2, SPAG1, ZMYND10 sont requis pour l'assemblage des bras de dynéine de l'axonème; DNAAFl, DNAH5, DNAL1, DNAIl/2, DNAL1 sont des composants des bras de dynéine; DRCl est un composant du complexe régulateur nexine/dynéine; LRRC6, 0FD1, RPGR, MKS1/3/5 interviennent dans la formation du centriole basal ; RSPHI/4A/9, RPGRIPl sont des composants de l'axonème ; $E V C l / 2$ sont des partenaires dominants négatifs du co-récepteur Smoothened; PKD2 est un canal calcium; PKD1 est un mécanorécepteur; MKKS, BBS12 sont des protéines chaperones ; BBS1/2/4/5/7/8/9/17/18 forment le BBSome qui recrute la GTPase Rab8A pour le transport vésiculaire vers le cil; BBS3/14 sont requis pour le transport vésiculaire; BBS11 est une ubiquitine ligase $\varepsilon 3$; IFT88, BBS19 sont des composants du transport intraflagellaire ; BBS15 est impliqué dans la polarité planaire. ARMC4 : armadillo repeat containing 4; C210RF59 : chromosome 21 open reading frame 59 ; CCDC39: coiled-coil domain containing 39 ; DNAAF1 : dynein, axonemal, assembly factor 1 ; DNAH5 : dynein, axonemal, heavy chain 5 ; DNAll : dynein, axonemal, intermediate chain 1 ; DNAL1 : dynein, axonemal, light chain 1 ; DRCl : dynein regulatory complex subunit 1 ; DYXICl : dyslexia susceptibility 1 candidate 1 ; HEATR2 : HEAT repeat containing 2 ; HYDIN : hydrocephalus-inducing protein ; LRRC6 : leucine rich repeat containing 6 ; 0FD1 : oral-facial-digital syndrome 1 ; RPGR : retinitis pigmentosa GTPase regulator; RSPHI : radial spoke head 1 homolog ; SPAGl : sperm-associated antigen 1 ; ZMYND10 : zinc finger, MYND-type containing 10.

Chez l'embryon, une plus forte proportion de cellules ciliées a été décrite parmi les cellules musculaires (cardiomyocytes) peu matures, dont les cils sont souvent invaginés dans une poche ciliaire $[8,9]$. $\varepsilon$ n accord avec cette observation, les cils sont détectés dans le cœur embryonnaire entre 9,5 jours $(\varepsilon 9,5)$ et 12,5 jours $(\varepsilon 12,5)$ de développement chez la souris $[10,11]$, alors que, chez l'adulte, ils sont détectés dans les cellules interstitielles non musculaires [9]. À quel stade les cils sont-ils perdus dans les cardiomyocytes? Cette perte est-elle irréversible? Ces questions restent encore ouvertes. Chez l'embryon, les cardiomyocytes des oreillettes et de la base des ventricules sont plus fréquemment ciliés [10]. Dans l'épicarde embryonnaire, la tubuline acétylée, associée aux microtubules stabilisés et présente notamment dans les cils, est enrichie du côté de la cavité péricardique [11]. Dans l'endocarde embryonnaire, les cils font saillie dans la cavité, en contact direct avec le flux sanguin, et sont localisés préférentiellement dans les zones soumises à un moindre stress mécanique, comme 


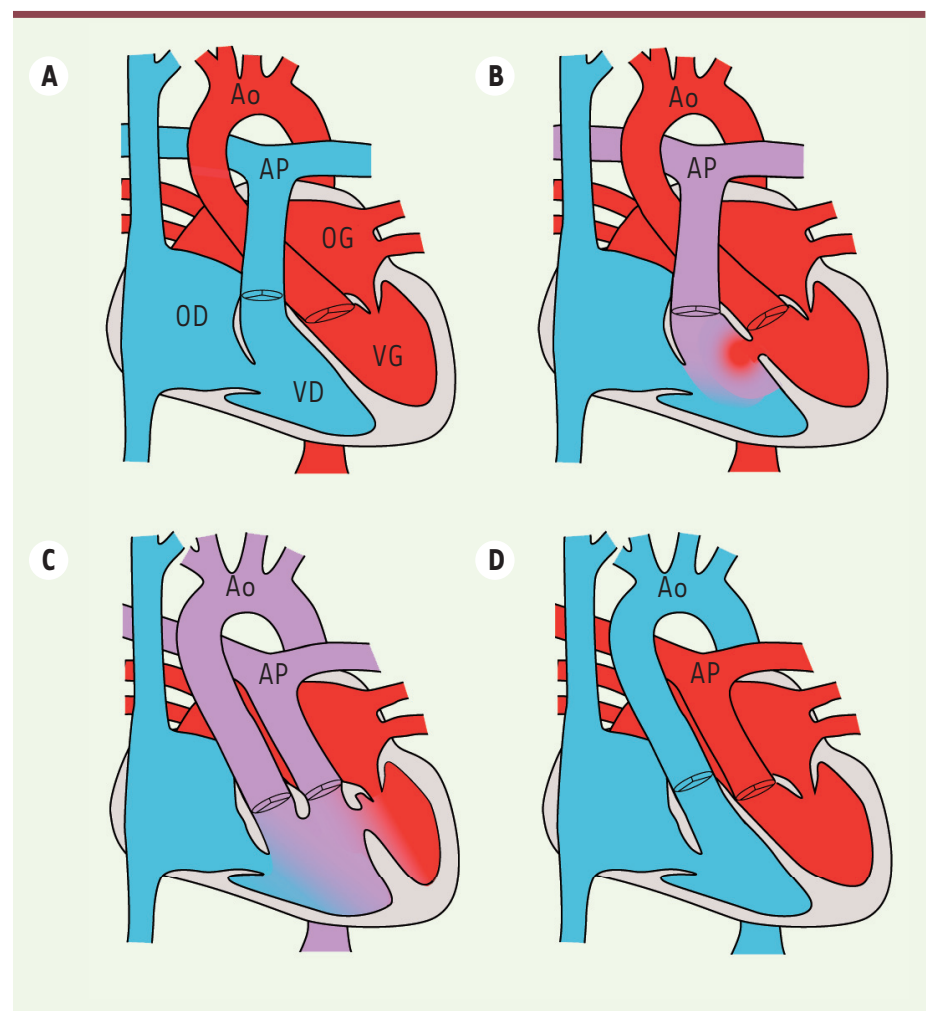

les oreillettes, la base des trabéculations du ventricule ou la base des bourgeons des valves atrio-ventriculaires. Cette régionalisation suggère que le cil primaire peut jouer un rôle de capteur de forces hémodynamiques [12]. Des cils ont également été observés dans le mésenchyme des bourgeons de valves; ils n'ont pas d'orientation apparente [11], et contiennent des antigènes spécifiques tels que la petite GTPase ARL13B (ADP-ribosylation factor-like 13B) [10]. Dans les muscles lisses des vaisseaux du cœur, la présence de cils primaires n'a pas encore été rapportée, alors qu'ils jouent un rôle dans d'autres vaisseaux [13]. Ainsi, par sa large distribution dans le cœur embryonnaire, le cil primaire se positionne comme un acteur important dans différents processus de la morphogenèse cardiaque.

\section{Signalisation droite/gauche et alignement des chambres cardiaques}

Les cils motiles et primaires sont essentiels dans le nœud, une structure embryonnaire qui est le siège de la mise en place de la latéralité droite/gauche de l'organisme (Figure 1A-B) [6, 14]. Cette latéralité a plusieurs conséquences sur la morphogenèse du cœur. Le cœur se forme à partir de précurseurs localisés de part et d'autre de la ligne médiane, recevant ainsi des signaux droits ou gauches (Figure 3 , $\varepsilon 6,5)$. Ainsi, les oreillettes droite et gauche diffèrent par leur morphologie, mais aussi par le drainage veineux et la nature des valves conduisant au ventricule adjacent. La signalisation droite/gauche est aussi requise pour des mouvements de courbure du cœur embryonnaire (Figure $3, \varepsilon 8,5$ ), qui conduisent au positionnement relatif des ventricules et des troncs artériels, ainsi qu'au positionnement du cœur
Figure 2. Exemples de malformations cardiaques associées à des ciliopathies. A. Dans un cœur normal, une double circulation sanguine est établie, qui sépare le sang carboné (bleu) du sang oxygéné (rouge). AP : artère pulmonaire; Ao: aorte; $O D$ : oreillette droite; $O G$ : oreillette gauche; VD: ventricule droit; VG : ventricule gauche. $B$. Dans le cas d'un défaut de cloisonnement ventriculaire, un mélange des sangs se produit (violet), avec des conséquences sur la pression sanguine dans le cœur. C. Dans un ventricule droit à double issue, les deux artères (aorte et artère pulmonaire) débouchent du ventricule droit, ce qui entrave l'oxygénation du sang distribué aux organes. D. La transposition des gros vaisseaux correspond à une inversion des artères, ce qui empêche le sang oxygéné d'arriver aux organes. Ces malformations doivent être réparées à la naissance.

à gauche de la cage thoracique. Ainsi, la structure du cœur est particulièrement sensible aux perturbations dans la détermination de la droite et de la gauche de l'embryon, ce qui a des conséquences sur la mise en place de la double circulation sanguine (pulmonaire et systémique).

Dans plusieurs modèles animaux, l'absence ou le dysfonctionnement des cils affectent la morphogenèse du cœur. L'absence de cils, résultant d'un défaut $d u$ centriole basal chez les mutants $0 \mathrm{fdl}^{-/-}$(oral-facialdigital syndrome 1) [15] ou de transport ciliaire dans les mutants $1 \mathrm{ft}^{88^{-1-}}$ (intraflagellar transport 88 homo$\log ), 1 \mathrm{ft} 172^{-/-}$et $\mathrm{Kif3a}^{-/-}$(kinesin $(\rightarrow)$ Voir la Synthèse family member 3A) [16, 17, 45] de C. Fort et P. Bastin, $\rightarrow$ induit une détermination bila- page 955 de ce numéro térale de la gauche de l'embryon, la courbure du cœur se faisant alors au hasard vers la droite ou la gauche de l'embryon. Parfois, la ciliogenèse est partielle, avec des cils plus courts ou moins nombreux. On observe, par exemple, des défauts de cloisonnement de la voie efférente et des ventricules chez le mutant Fuz (fuzzy planar cell polarity protein), qui comporte une mutation dans un effecteur de la polarité planaire ${ }^{1}$ impliqué dans le trafic membranaire [18], alors que chez le mutant Mks 1 (Meckel syndrome, type 1), que caractérise une altération d'un régulateur du centriole basal, la courbure du cœur se fait au hasard, et, plus tard, une transposition des gros vaisseaux apparaît [19]. L'invalidation du gène Pifo (Pitchfork) induit des cils anormaux, dupliqués, et aboutit à un ventricule droit à double issue, malformation dans laquelle l'aorte et l'artère pulmonaire sortent toutes deux du ventricule droit [20]. L'invalidation de la dynéine axonémale Dnaicl (dynein,

${ }^{1}$ La polarité planaire oriente les cellules épithéliales en surface, à la différence de la polarité apico-basale selon un axe perpendiculaire. 

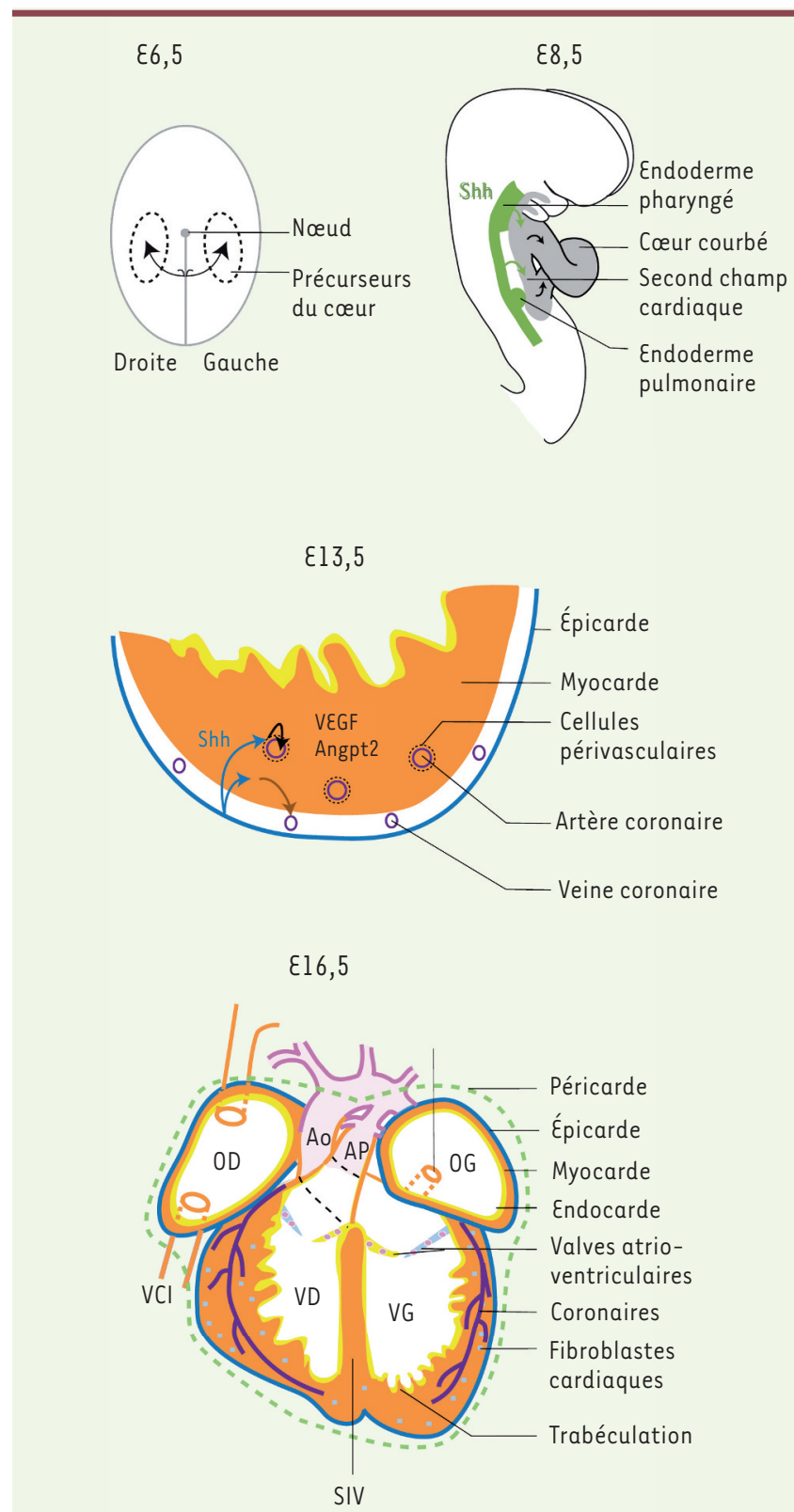

axonemal, intermediate chain 1), qui n'affecte pas la ciliogenèse mais la motilité des cils, conduit à des défauts de la courbure du cœur, se traduisant par un mauvais positionnement du cœur si la courbure est inversée, ou à une hétérotaxie si la courbure est incomplète [21]. Quand la kinase ciliaire Nek8 (NIMA-related kinase 8) est mutée, les cils se forment normalement. Cependant, l'orientation de la courbure du cœur se fait au hasard chez ces mutants, qui présentent aussi des défauts de cloisonnement des chambres cardiaques et un ventricule droit à double issue [22].

Dans ces modèles animaux, l'inactivation génétique est zygotique, c'est-à-dire ubiquitaire, ce qui ne permet pas de comprendre dans quels types cellulaires les cils sont requis. II est très probable que
Figure 3. Rôle des cils à différentes étapes de la formation du cœur. $\varepsilon 6,5$ ( 6,5 jours après la fécondation) : au moment de la gastrulation, les cils du nœud sont importants pour la détermination de la droite et de la gauche de l'embryon. Les flèches noires indiquent la migration des précurseurs cardiaques de part et d'autre de la ligne primitive (grise) où a lieu la gastrulation. ع8,5: la courbure du cœur est sensible à la latéralité droite/gauche. Par ailleurs, la sécrétion de Sonic hedghog (Shh) dans l'endoderme active (flèches vertes) une voie de signalisation dans les précurseurs du second champ cardiaque pour le cloisonnement des oreillettes et des gros vaisseaux artériels. Les flèches noires indiquent le recrutement des précurseurs cardiaques dans le cœur. $\varepsilon 13,5$ : schéma d'une coupe de ventricule représentant les différents tissus cardiaques. Shh est ici sécrété par l'épicarde (flèches bleues), et est nécessaire dans les cellules périvasculaires (en noir) et du myocarde (orange) pour stimuler la formation du réseau coronaire via la sécrétion de facteurs de croissance vasculaire endothélial (VEGF) et d'angiopoïétine (Angpt2). ع16,5 : schéma du cœur mature montrant l'organisation des différents tissus cardiaques, ainsi que l'alignement des chambres cardiaques et des gros vaisseaux. $A P$ : artère pulmonaire ; $A 0$ : aorte ; $O D$ : oreillette droite ; $O G$ : oreillette gauche; SIV : septum interventriculaire; $\mathrm{VCI}$ : veine cave inférieure; VCS : veine cave supérieure; VD: ventricule droit ; VG : ventricule gauche; VP : veine pulmonaire.

les phénotypes de courbure du cœur ou d'hétérotaxie résultent d'un dysfonctionnement ciliaire indirect, par l'intermédiaire de la signalisation droite/gauche, dans les cellules du nœud qui ne participent pas au cœur. Outre ce rôle, le cil est nécessaire à la transduction de voies de signalisation dans des cellules qui colonisent le cœur.

\section{Signalisation Hedgehog}

La voie de signalisation Hedgehog (hh), qui régule la croissance et la détermination des tissus, dépend du cil primaire $[17,23]$. En présence d'un ligand (Shh, Sonic hedgehog, Ihh, Indian hedgehog ou Dhh, Desert hedgehog), le co-récepteur Smoothened est concentré au niveau du cil, où il peut déclencher l'activation des facteurs de transcription Gli (Figure 1C). Shh et lhh jouent un rôle précoce dans la détermination de la droite et de la gauche de l'embryon [24], alors que Smoothened et Ihh sont essentiels à la mise en place de la circulation sanguine par la vascularisation du sac vitellin [25]. Ces deux processus ont des répercussions indirectes sur la formation du cœur. De manière directe, Shh est requis pour le cloisonnement du cœur et la formation des coronaires, les vaisseaux qui irriguent le muscle cardiaque. 


\section{Cloisonnement du cœur}

Pour le bon fonctionnement du cœur, il est crucial que le sang oxygéné dans les poumons ne se mélange pas au sang carboné qui revient des autres organes. Ainsi, dans le cœur embryonnaire qui est un tube, les chambres cardiaques (oreillettes, ventricules) et les gros vaisseaux (artère pulmonaire, aorte) doivent être cloisonnés (Figure 2). Parmi les cellules qui participent à ce processus, il y a des précurseurs mésodermiques (Figure $3, \varepsilon 8,5-\varepsilon 10,5$ ) qui expriment le récepteur Patched-1 ou le facteur de transcription Glil, ce qui indique que ces cellules sont sensibles à la sécrétion de Shh par les cellules voisines de l'endoderme de l'intestin antérieur [26, 27]. Par traçage génétique avec une recombinase Cre inductible dont l'expression dépend des séquences régulatrices de Glil, il a été montré que ces cellules participent à la cloison des oreillettes et à l'artère pulmonaire [26]. Ainsi, en présence d'une mutation de Shh ubiquitaire [28] ou de celle du co-récepteur Smoothened spécifiquement dans les cellules précurseurs du second champ cardiaque $[27,29]$, la cloison des oreillettes ne se forme pas, l'artère pulmonaire est atrophiée et le cloisonnement des ventricules est incomplet. Ces animaux ne survivent pas après la naissance, en raison de l'impossibilité d'apporter aux organes un sang suffisamment oxygéné. L'action de Shh est nécessaire à la survie [28] ou à la prolifération [30] cellulaires, ou bien elle module la différenciation musculaire $[24,27,31]$.

Puisque Sh a besoin du cil primaire pour activer sa voie de signalisation, les mutations qui affectent la ciliogenèse affectent le cloisonnement du cœur. C'est le cas chez les mutants hypomorphes Ift $88^{c b s / c b s}$, qui reproduisent les défauts d'une faible signalisation Shh [32]. La signalisation Shh est modulée par d'autres composants de la machinerie de transport ciliaire, tels que Ift25 [33], qui sont ainsi requis pour le cloisonnement du cœur. Chez l'homme, de tels défauts sont observés dans le syndrome ciliopathique Ellis van Creveld (EVC). Les gènes responsables de la maladie, $\varepsilon V C$ et $\varepsilon V C 2$, codent pour des partenaires dominants négatifs du co-récepteur Smoothened dont la localisation est régulée par un complexe ciliaire récemment identifié

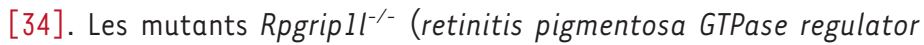
interacting protein 11 ), qui ont des cils plus courts, ont des défauts de maturation de Gli3 associés à des défauts de cloisonnement des ventricules. Un autre aspect de ces mutants, l'amincissement des parois des chambres cardiaques qui est associé à une baisse de prolifération cellulaire, suggère que la signalisation Shh pourrait jouer un autre rôle, par exemple dans les cellules des chambres cardiaques. Ainsi, l'effecteur Gli3 de la signalisation Shh a été observé dans les cils primaires des ventricules embryonnaires [10].

\section{Formation des coronaires}

Lorsque le muscle cardiaque (myocarde) s'épaissit, il doit être irrigué par un réseau de vaisseaux sanguins, les coronaires. La voie Shh est nécessaire à la formation de ce réseau et implique une signalisation entre différents tissus cardiaques. Shh est exprimé dans l'épicarde, le tissu qui recouvre le myocarde, en une vague qui part de la base des ventricules à $\varepsilon 12,5$ jusqu'à l'apex à $\varepsilon 13,5$ [35]. La mutation du co-récepteur Smoothened, spécifiquement dans le myocarde, arrête la croissance des veines coronaires, tandis que la même mutation, ciblée dans les cellules périvasculaires, arrête la croissance des artères coronaires [36]. Ainsi, la sécrétion de Shh par les cellules de l'épicarde est détectée par les cellules voisines, myocardiques ou périvasculaires (Figure 3, ع13,5) qui, en réponse, expriment des facteurs de croissance de l'endothélium vasculaire (VEGF [vascular endothelial growth factor], Angpt2 [angiopoietin 2]). Cependant, des défauts des vaisseaux coronaires n'ont pas encore été décrits dans des mutants ciliaires ou dans des ciliopathies; il serait intéressant de rechercher si ces défauts existent.

Nous avons vu que la transduction de la voie Hedgehog est importante dans des cellules qui colonisent le cœur. Il est vraisemblable que cette transduction passe par le cil primaire, puisque des mutations dans des composants ciliaires conduisent à des phénotypes similaires. Un autre exemple de voie de signalisation qui passe par le cil primaire et est importante pour la formation du cœur, est celui des polycystines.

\section{Polycystines}

Les polycystines sont des protéines membranaires souvent localisées dans le cil primaire (Figure 1B). Chez l'homme, des mutations des polycystines sont responsables de la polykystose rénale autosomique dominante (PKD). PKD2 agit comme un canal calcium, tandis que PKDl agit comme un mécanorécepteur des interactions cellule-cellule ou cellule-matrice extracellulaire [37]. Chez la souris, la mutation des gènes $P k d l$ et $P k d 2$ conduit à des malformations des valves cardiaques [38], ainsi qu'à des défauts de cloisonnement des chambres cardiaques [38, 39]. Dans le cas d'une perte de fonction, un myocarde plus fin a été observé $[11,38]$, alors que la surexpression de $P k d l$ (entraînant un niveau 2 à 15 fois supérieur au niveau normal) conduit à un effet inverse d'hypertrophie du myocarde [40], ce qui rappelle la maladie humaine. Lorsque la mutation de $P k d l$ est restreinte aux cellules endothéliales [41] ou aux muscles lisses et cardiaque [42], elle ne cause pas de défaut cardiaque, à la différence de la mutation ubiquitaire. L'interprétation de cette différence n'est pas claire : elle pourrait être due à une variation dans la séquence de la mutation ou à l'intervention de Pkdl dans un autre type cellulaire que les cellules endothéliales des muscles lisses et cardiaque, comme par exemple les cellules interstitielles du cœur. Ainsi, le mécanisme d'action de Pkdl et Pkd2 dans le cœur reste à déterminer. L'effet est-il direct dans des cellules cardiaques? Quel compartiment cellulaire est contrôlé et par quelle cascade de 
signalisation? De même, le lien entre Pkd et cil cardiaque n'a pas été démontré.

D'autres voies de signalisation ont été reliées au cil primaire, comme par exemple la voie Wnt canonique, la voie Wnt de la polarité planaire, ainsi que les voies PDGFR $\alpha$ (plateled-derived growth factor receptor $\alpha$ ) [1] et TGF $\beta$ (transforming growth factor $\beta$ ) [43], qui sont importantes pour certaines étapes de la formation du cœur. Cependant, les mutants de composants ciliaires conduisent à des phénotypes moins sévères que les mutants des voies Wnt et TGF $\beta$, ce qui indique que le cil primaire n'est pas toujours requis pour ces voies. Le rôle de ces voies dans les cils des cellules cardiaques in vivo reste donc à démontrer.

\section{Conclusions}

Par leur large distribution dans de nombreux types cellulaires, les cils primaires jouent un rôle important dans la formation des organes, y compris le cœur. Le dysfonctionnement des cils primaires ou des voies de signalisation dont la transduction se produit habituellement dans le cil primaire conduit à un large spectre de malformations cardiaques. Le cil est un organite spécialisé dont la structure et les capacités sensorielles varient d'un type cellulaire à un autre $[44,45]$. II est possible que les différents types de malformations cardiaques résultent d'une fonction ciliaire spécifique. Cependant, les modèles actuels résultent d'inactivations ubiquitaires de gènes ciliaires. Le rôle des cils doit maintenant être disséqué plus finement par un contrôle spatio-temporel des mutations ciliaires, pour découpler les différents phénotypes et identifier le rôle des cils d'un type cellulaire spécifique à un stade donné de développement. En outre, ces mutations contrôlées pourraient permettre de révéler d'autres fonctions des cils primaires, qui sont des structures dynamiques au cours du cycle cellulaire, comme par exemple dans le contrôle de la prolifération cellulaire [49] $(\rightarrow)$, de la différenciation cellulaire, ou de l'orientation de la division cellulaire $[50](\rightarrow)$.

Outre leur intérêt pour déchiffrer les mécanismes fondamentaux de la morphogenèse du cœur, ces études seront indispensables pour mieux comprendre l'étiologie des cardiopathies associées $(\rightarrow)$ Voir la Synthèse de N. Delgehyr et N. Spassky, page 976 de ce numéro

\section{$(\rightarrow)$ Voir la Synthèse de N. Taulet et B. Delaval, page 1040 de ce numéro} aux ciliopathies. $\diamond$

\section{SUMMARY}

\section{Cilia and heart morphogenesis}

After the seminal discovery in 2000 that primary cilia are functional organelles which are essential for embryonic development, several mouse models of ciliopathies have been generated. The heart is frequently affected, with a large spectrum of malformations. The cilia of the node are required early in development in the determination of the left/right laterality of the embryo, which has secondary consequences on the formation of the heart. Thus, abnormal looping of the heart is a recurrent phenotype in models of ciliopathies. However, the function of primary cilia in cardiac cells remains poorly understood. Receptors such as polycystins or hedgehog receptors are usually localized in the primary cilium, raising the possibility that these signalling pathways, which are important for the septation and the growth of the heart, are transduced in primary cilia of cardiac cells. Knowledge of the roles of primary cilia at different steps of heart development and in different cardiac cell types will be essential to better understand the origin of human cardiopathies associated with ciliopathies. $\diamond$

\section{LIENS D'INTÉRÊT}

Les auteurs déclarent n'avoir aucun lien d'intérêt concernant les données publiées dans cet article.

\section{REMERCIEMENTS}

Les travaux de l'équipe de S. Meilhac sont financés par la Fondation pour la recherche médicale, l'Agence nationale pour la recherche et l'Institut national de la santé et de la recherche médicale.

\section{RÉFÉRENCES}

1. Goetz SC, Anderson KV. The primary cilium: a signalling centre during vertebrate development. Nat Rev Genet 2010 ; 11 : 331-44.

2. Pazour GJ, Dickert BL, Vucica Y, et al. Chlamydomonas IFT88 and its mouse homologue, polycystic kidney disease gene $\operatorname{tg} 737$, are required for assembly of cilia and flagella. J Cell Biol 2000 ; 151 : 709-18.

3. Baker K, Beales PL. Making sense of cilia in disease: the human ciliopathies. Am J Med Genet C Semin Med Genet 2009 ; 151C : 281-95.

4. Shenje LT, Andersen P, Halushka MK, et al. Mutations in Alstrom protein impair terminal differentiation of cardiomyocytes. Nat Commun 2014 $5: 3416$.

5. Tobin JL, Beales PL. The nonmotile ciliopathies. Genet Med 2009; 11 : 386-402.

6. Nonaka S, Tanaka Y, Okada Y, et al. Randomization of left-right asymmetry due to loss of nodal cilia generating leftward flow of extraembryonic fluid in mice lacking KIF3B motor protein. Cell 1998 ; 95 : 829-37.

7. Brueckner M. Heterotaxia, congenital heart disease, and primary ciliary dyskinesia. Circulation 2007 ; 115 : 2793-5.

8. Rash JE, Shay JW, Biesele JJ. Cilia in cardiac differentiation. J Ultrastruct Res $1969 ; 29: 470-84$.

9. Myklebust R, Engedal H, Saetersdal TS, Ulstein M. Primary 9+0 cilia in the embryonic and the adult human heart. Anat Embryol 1977 ; 151 : 127-39.

10. Gerhardt C, Lier JM, Kuschel S, Rüther U. The ciliary protein Ftm is required for ventricular wall and septal development. PLoS One 2013 ; 8 : e57545.

11. Slough J, Cooney L, Brueckner M. Monocilia in the embryonic mouse heart suggest a direct role for cilia in cardiac morphogenesis. Dev Dyn 2008; 237 : 2304-14

12. Van der Heiden K, Groenendijk BC, Hierck BP, et al. Monocilia on chicken embryonic endocardium in low shear stress areas. Dev Dyn $2006 ; 235$ : 19-28.

13. Lu CJ, Du H, Wu J, et al. Non-random distribution and sensory functions of primary cilia in vascular smooth muscle cells. Kidney Blood Press Res 2008 $31: 171-84$.

14. Babu D, Roy S. Left-right asymmetry: cilia stir up new surprises in the node. Open Biol 2013 ; 3 : 130052.

15. Ferrante MI, Zullo A, Barra A, et al. Oral-facial-digital type I protein is required for primary cilia formation and left-right axis specification. Nat Genet 2006 ; $38: 112-7$.

16. Takeda S, Yonekawa $Y$, Tanaka $Y$, et al. Left-right asymmetry and kinesin superfamily protein KIF3A: new insights in determination of laterality and mesoderm induction by kif3A-/- mice analysis. J Cell Biol 1999 ; 145 : 825-36.

17. Huangfu D, Liu A, Rakeman AS, et al. Hedgehog signalling in the mouse requires intraflagellar transport proteins. Nature $2003 ; 426: 83-7$.

18. Gray RS, Abitua PB, Wlodarczyk BJ, et al. The planar cell polarity effector Fuz is essential for targeted membrane trafficking, ciliogenesis and mouse embryonic development. Nat Cell Biol 2009 ; 11 : 1225-32. 


\section{RÉFÉRENCES}

19. Cui C, Chatterjee B, Francis D, et al. Disruption of Mksl localization to the mother centriole causes cilia defects and developmental malformations in Meckel-Gruber syndrome. Dis Model Mech $2011 ; 4: 43-56$

20. Kinzel $D$, Boldt K, Davis $\varepsilon \varepsilon$, et al. Pitchfork regulates primary cilia disassembly and left-right asymmetry. Dev Cell $2010 ; 19: 66-77$

21. Francis RJ, Christopher A, Devine WA, et al. Congenital heart disease and the specification of leftright asymmetry. Am J Physiol Heart Circ Physiol 2012 ; 302 : H2102-11.

22. Manning DK, Sergeev M, van Heesbeen RG, et al. Loss of the ciliary kinase Nek8 causes left-right asymmetry defects. J Am Soc Nephrol $2013 ; 24: 100-12$

23. Nozawa YI, Lin C, Chuang PT. Hedgehog signaling from the primary cilium to the nucleus: an emerging picture of ciliary localization, trafficking and transduction. Curr Opin Genet Dev $2013 ; 23: 429-37$.

24. Zhang XM, Ramalho-Santos M, McMahon AP. Smoothened mutants reveal redundant roles for Shh and Ihh signaling including regulation of L/R symmetry by the mouse node. Cell $2001 ; 106$ : 781-92.

25. Byrd N, Becker S, Maye P, et al. Hedgehog is required for murine yolk sac angiogenesis. Development $2002 ; 129$ : 361-72.

26. Hoffmann AD, Peterson MA, Friedland-Little JM, et al. Sonic hedgehog is required in pulmonary endoderm for atrial septation. Development 2009; 136 : 1761-70.

27. Goddeeris MM, Rho S, Petiet $A$, et al. Intracardiac septation requires hedgehog-dependent cellular contributions from outside the heart. Development 2008 ; 135 : 1887-95.

28. Washington Smoak I, Byrd NA, Abu-Issa R, et al. Sonic hedgehog is required for cardiac outflow tract and neural crest cell development. Dev Biol $2005 ; 283: 357-72$.

29. Lin L, Bu L, Cai CL, et al. Isll is upstream of sonic hedgehog in a pathway required for cardiac morphogenesis. Dev Biol $2006 ; 295$ : 756-63.

30. Dyer LA, Kirby ML. Sonic hedgehog maintains proliferation in secondary heart field progenitors and is required for normal arterial pole formation. Dev Biol 2009 ; 330 : 305-17.

31. Clement CA, Kristensen SG, Møllgård K, et al. The primary cilium coordinates early cardiogenesis and hedgehog signaling in cardiomyocyte differentiation. J Cell Sci 2009; $122: 3070-82$.

32. Willaredt MA, Gorgas K, Gardner HA, Tucker KL. Multiple essential roles for primary cilia in heart development. Cilia $2012 ; 1: 23$.

33. Keady BT, Samtani R, Tobita K, et al. IFT25 links the signal-dependent movement of Hedgehog components to intraflagellar transport. Dev Cell $2012 ; 22: 940-51$.

34. Pusapati GV, Hughes CE, Dorn KV, et al. EFCAB7 and IQCE regulate hedgehog signaling by tethering the EVC-EVC2 complex to the base of primary cilia. Dev Cell $2014 ; 28: 483-96$.

35. Lavine KJ, White AC, Park C, et al. Fibroblast growth factor signals regulate a wave of Hedgehog activation that is essential for coronary vascular development. Genes Dev $2006 ; 20: 1651-66$.
36. Lavine KJ, Schmid GJ, Smith CS, Ornitz DM. Novel tool to suppress cell proliferation in vivo demonstrates that myocardial and coronary vascular growth represent distinct developmental programs. Dev Dyn 2008 ; 237 : 713-24.

37. Zhou J. Polycystins and primary cilia: primers for cell cycle progression. Annu Rev Physiol $2009 ; 71: 83-113$.

38. Boulter C, Mulroy S, Webb S, et al. Cardiovascular, skeletal, and renal defects in mice with a targeted disruption of the Pkdl gene. Proc Natl Acad Sci USA $2001 ; 98$ : 12174-9.

39. Wu G, Markowitz GS, Li L, et al. Cardiac defects and renal failure in mice with targeted mutations in Pkd2. Nat Genet $2000 ; 24: 75-8$.

40. Kurbegovic A, Côté 0 , Couillard M, et al. Pkdl transgenic mice: adult model of polycystic kidney disease with extrarenal and renal phenotypes. Hum Mol Genet $2010 ; 19$ : 1174-89.

41. Garcia-Gonzalez MA, Outeda P, Zhou $Q$, et al. Pkdl and Pkd2 are required for normal placental development. PLoS One 2010 ; 5 : e12821.

42. Hassane $\mathrm{S}$, Claij $\mathrm{N}$, Jodar $\mathrm{M}$, et al. Pkdl-inactivation in vascular smooth muscle cells and adaptation to hypertension. Lab Invest $2011 ; 91: 24-32$.

43. Clement CA, Ajbro KD, Koefoed K, et al. TGF-beta signaling is associated with endocytosis at the pocket region of the primary cilium. Cell Rep 2013; 3: 1806-14.

44. Choksi SP, Lauter G, Swoboda P, Roy S. Switching on cilia: transcriptional networks regulating ciliogenesis. Development 2014 ; 141 : 1427-41.

45. Fort $C$, Bastin P. Élongation de l'axonème et dynamique du transport intraflagellaire. Med Sci (Paris) 2014 ; 30 : 955-61.

46. Bachmann-Gagescu R. Complexité génétique des ciliopathies et identification de nouveaux gènes. Med Sci (Paris) $2014 ; 30$ : 1011-23.

47. Chennen K, Scerbo MJ, Dollfus $H$, et al. BBS : cils et obésité ; de la génétique à l'approche intégrative. Med Sci (Paris) 2014 ; 30 : 1034-9.

48. Pacès-Fessy M. Cils et kystes rénaux. Med Sci (Paris) 2014 ; 30 : 1024-33.

49. Delgehyr N, Spassky N. Cil primaire, cycle cellulaire et prolifération. Med Sci (Paris) $2014 ; 30: 976-9$.

50. Taulet N, Delaval B. De nouvelles fonctions extraciliaires pour les protéines ciliaires. Quelles conséquences sur l'apparition de ciliopathies? Med Sci (Paris) $2014 ; 30: 1040-50$

\section{TIRÉS À PART}

S.M. Meilhac

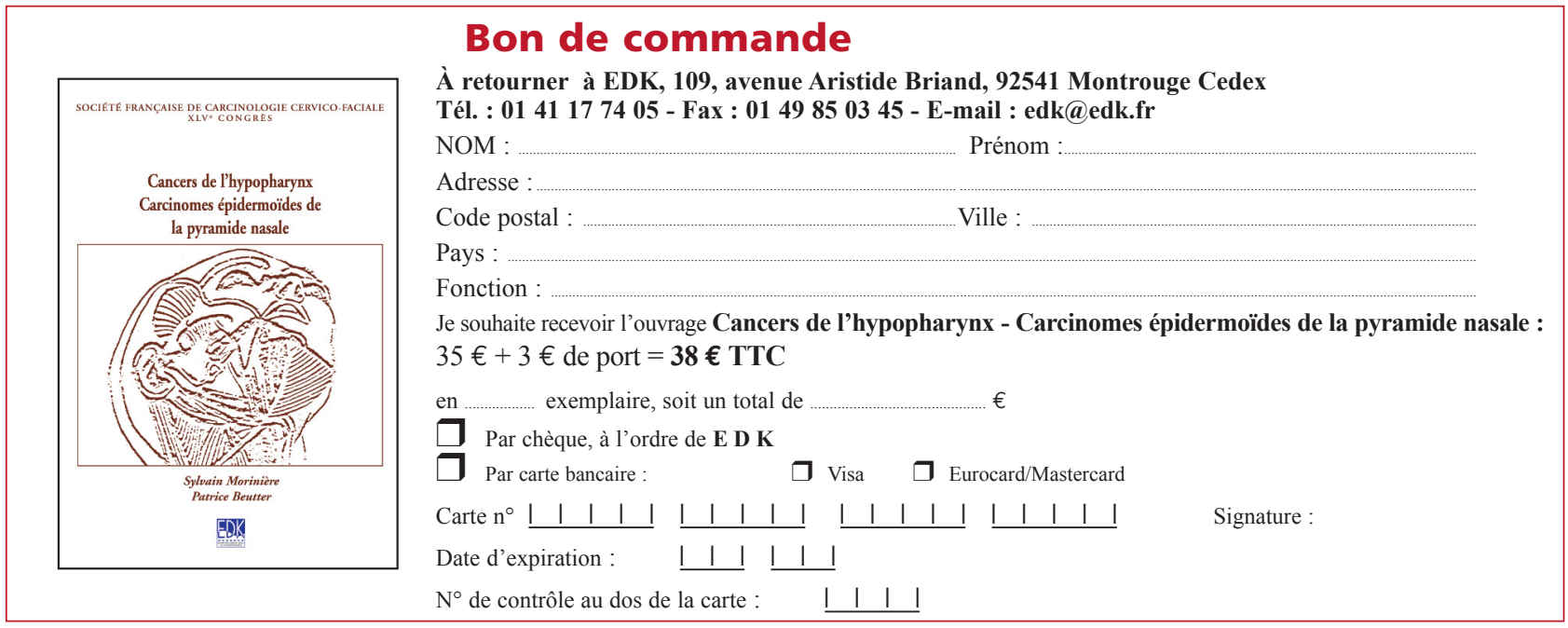

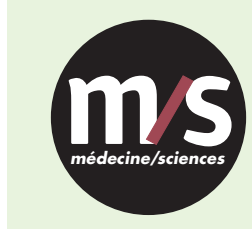

Tarifs d'abonnement $\mathrm{m} / \mathrm{s}-2014$

Abonnez-vous

à médecine/sciences
$>$ Grâce à $m / s$, vivez en direct les progrès des sciences biologiques et médicales

Bulletin d'abonnement page 1049 dans ce numéro de $\mathrm{m} / \mathrm{s}$

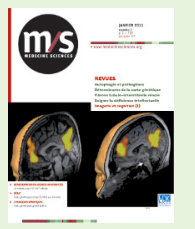

